J. Korean Math. Soc. 52 (2015), No. 4, pp. 751-763

http://dx.doi.org/10.4134/JKMS.2015.52.4.751

\title{
A CLASS OF NEW NEAR-PERFECT NUMBERS
}

\author{
YANBin Li AND QUNYing LiaO
}

\begin{abstract}
Let $\alpha$ be a positive integer, and let $p_{1}, p_{2}$ be two distinct prime numbers with $p_{1}<p_{2}$. By using elementary methods, we give two equivalent conditions of all even near-perfect numbers in the form $2^{\alpha} p_{1} p_{2}$ and $2^{\alpha} p_{1}^{2} p_{2}$, and obtain a lot of new near-perfect numbers which involve some special kinds of prime number pairs. One kind is exactly the new Mersenne conjecture's prime number pair. Another kind has the form $p_{1}=2^{\alpha+1}-1$ and $p_{2}=\frac{p_{1}^{2}+p_{1}+1}{3}$, where the former is a Mersenne prime and the latter's behavior is very much like a Fermat number.
\end{abstract}

\section{Introduction}

Definition 1.1. Let $n$ be a positive integer. Set $D=\{d: d \mid n, 1 \leqslant d \leqslant n\}$ and $\sigma(n)=\sum_{d \in D} d$.

(1) If $\sigma(n)=2 n$, then $n$ is called a perfect number.

(2) (Sierpinski) If there exists some $S \subseteq D-\{n\}$ such that $n=\sum_{d \in S} d$, then $n$ is called a pseudoperfect number.

(3) (Shevelev) If there exists some $d \in D-\{n\}$ such that $\sigma(n)=2 n+d$, then $n$ is called a near-perfect number with the redundant divisor $d$.

(4) (Pollack \& Shevelev) If there exist some $r, 0 \leqslant r \leqslant k$ and $S \subseteq D-\{n\}$ with $|S|=r$, such that $\sigma(n)=2 n+\sum_{d \in S} d$, then $n$ is called a $k$-nearperfect number with the redundant divisors set $S$.

By Definition 1.1 above, it is easy to see that pseudoperfect numbers are a generalization for perfect numbers, near-perfect numbers are special pseudoperfect numbers, and perfect numbers and near-perfect numbers constitute 1-near-perfect numbers.

People have been interested in perfect numbers for a long time. Euclid and Euler determined all even perfect numbers, which are closely related to

Received August 19, 2014; Revised February 23, 2015.

2010 Mathematics Subject Classification. 11A25, 11B83.

Key words and phrases. perfect number, pseudoperfect number, near-perfect number, $k$-near-perfect number.

The work is supported by the Natural Science Foundation of China with No. 11401408, Sichuan Province Foundation of China with No. 14ZA0034, and Sichuan Normal University Key Project Foundation with No. 13ZDL06. 
Mersenne primes. For odd perfect numbers, Euler obtained a necessary condition for the existence (see [5]). In recent years, there have been many papers for odd perfect numbers having to do with the conjecture that there exists no odd perfect numbers (see $[2,3,4,7,12]$ ). Until now, the conjecture has not been proved. Therefore, people study other similar numbers, such as pseudoperfect numbers, near-perfect numbers, $k$-near-perfect numbers and deficient-perfect numbers, which are closely related to perfect numbers (see $[8,9,10,13])$.

In 2012, based on the criterion for the existence of even perfect numbers, Paul Pollack and Vladimir Shevelev obtained 3 classes of even near-perfect numbers as follows (see [8]).

Proposition 1.2. Suppose that $2^{p}-1$ is a Mersenne prime. Then $n=$ $2^{p-1}\left(2^{p}-1\right)^{2}$ is a near-perfect number with the redundant divisor $2^{p}-1$.

Proposition 1.3. Let $t$ and $k$ be positive integers with $t \geqslant k+1$. Suppose that $2^{t}-2^{k}-1$ is an odd prime number. Then $n=2^{t-1}\left(2^{t}-2^{k}-1\right)$ is near-perfect with the redundant divisor $2^{k}$.

Proposition 1.4. Let $\alpha$ be a positive integer and let $p$ be a prime number. Suppose that $m$ is an even perfect number and $2^{p-1} \| m$. Then $n=2^{\alpha} m$ is near-perfect if and only if $\alpha=1$ or $\alpha=p$.

It is easy to see that the near-perfect numbers in Proposition 1.2-1.4 are in the form $2^{\alpha} p^{\beta}$, where $\alpha, \beta \geqslant 1$ and $p$ is an odd prime number. Noting that $40=2^{3} \cdot 5$ which isn't in Propositions $1.2-1.4$ is also a near-perfect number with the redundant divisor 10, Chen and Ren improved the above results by proving the following proposition in [9].

Proposition 1.5. Suppose that $n$ has exactly two distinct prime divisors. Then $n$ is near-perfect if and only if $n=40$ or $n$ is given by one of Propositions $1.2-$ 1.4 .

Moreover, for near-perfect numbers with at least three distinct prime divisors, there is the following conjecture in [9].

Conjecture 1.6. For any $k \geqslant 3$, there exists only finitely many near-perfect numbers with exactly $k$ distinct prime divisors.

In fact, for the generalized case, let $\alpha_{i}$ be positive integers and let $p_{i}$ be distinct primes, where $i=1,2, \ldots, r$. Suppose that $n=p_{1}^{\alpha_{1}} \cdots p_{r}^{\alpha_{r}}$ is nearperfect with the redundant divisor $d$, namely, $\sigma(n)=2 n+d$. Note that for any $i=1,2, \ldots, r$,

$$
\sigma\left(p_{i}^{\alpha_{i}}\right)=p_{i}^{\alpha_{i}}+p_{i}^{\alpha_{i}-1}+\cdots+1, \operatorname{gcd}\left(p_{i}^{\alpha_{i}}, \sigma\left(p_{i}^{\alpha_{i}}\right)\right)=1,
$$

and so

$$
\sigma(n)=\sum_{d \mid n} d=\prod_{i=1}^{r} \sum_{j=0}^{\alpha_{i}} p_{i}^{j}=\prod_{i=1}^{r} \sigma\left(p_{i}^{\alpha_{i}}\right)=\sigma\left(p_{i}^{\alpha_{i}}\right) \sigma\left(\frac{n}{p_{i}^{\alpha_{i}}}\right) .
$$

Therefore we have the following lemma. 
Lemma 1.7. The assumptions are as the above. Then for any $i=1,2, \ldots, r$, we have $p_{i} \mid \sigma\left(n / p_{i}^{\alpha_{i}}\right)$ if $p_{i} \mid d$, or $p_{i} \mid\left(\sigma\left(n / p_{i}^{\alpha_{i}}\right)-d\right)$ if $p_{i} \nmid d$.

In particular, for an even near-perfect number $n$, from Lemma 1.7, we can get

Lemma 1.8. Let $r, \alpha_{i}$ be positive integers, and let $p_{i}$ be distinct primes $(1 \leqslant$ $i \leqslant r)$. Suppose that $n=2^{\alpha_{0}} p_{1}^{\alpha_{1}} \cdots p_{r}^{\alpha_{r}}$ is an even near-perfect number with the redundant divisor $d$. Then there exists some $i$ with $1 \leqslant i \leqslant r$, such that $\alpha_{i}$ is odd if and only if $2 \mid d$.

Proof. From $\sigma\left(n / 2^{\alpha_{0}}\right)=\prod_{i=1}^{r} \sum_{j=0}^{\alpha_{i}} p_{i}^{j}$ and all $p_{i}(1 \leqslant i \leqslant r)$ are odd primes, we know that there exists some $i \in\{1, \ldots, r\}$ such that $\alpha_{i}$ is odd if and only if $2 \mid \sigma\left(n / 2^{\alpha_{0}}\right)$.

On the one hand, from $2 \mid d$ and Lemma 1.7, we can get $2 \mid \sigma\left(n / 2^{\alpha_{0}}\right)$, which means that there exists some $i$ with $1 \leqslant i \leqslant r$ such that $\alpha_{i}$ is odd.

On the other hand, suppose that there exists some $i$ with $1 \leqslant i \leqslant r$ such that $\alpha_{i}$ is odd, then $2 \mid \sigma\left(n / 2^{\alpha_{0}}\right)$. In this case, if $2 \nmid d$, then by Lemma 1.7 we have $2 \mid\left(\sigma\left(n / 2^{\alpha_{0}}\right)-d\right)$. Thus from $2 \mid \sigma\left(n / 2^{\alpha_{0}}\right)$, we have $2 \mid d$, which is a contradiction.

Thus we complete the proof of Lemma 1.8.

Beyond that, in [8], the authors generalized near-perfect numbers to $k$-nearperfect numbers and proved the following proposition.

Proposition 1.9. Let $k \geqslant 2$ and $\alpha+1>r_{1}>r_{2}>\cdots>r_{k} \geqslant 1$. Suppose that $p=2^{\alpha+1}-2^{r_{1}}-\cdots-2^{r_{k}}-1$ is an odd prime number. Then $n=2^{\alpha} p$ is a $k$-near-perfect number with $k$ redundant divisors $2^{r_{1}}, \ldots, 2^{r_{k}}$.

In fact, some $k$-near-perfect numbers in Proposition 1.9 are also near-perfect. Using Proposition 1.5, one can deduce the following conclusion.

Corollary 1.10. Let $k \geqslant 2$ and $\alpha+1>r_{1}>r_{2}>\cdots>r_{k} \geqslant 1$. Suppose that $p=2^{\alpha+1}-2^{r_{1}}-\cdots-2^{r_{k}}-1$ is an odd prime number. Then the $k$-near-perfect number $n=2^{\alpha} p$ is also near-perfect if and only if one of the following is true.

(1) $k$ is a prime number, $\alpha=2 k-1$ and $r_{i}=2 k-i(1 \leqslant i \leqslant k)$.

(2) $n=40$.

Proof. From the definition of near-perfect numbers, the sufficiency is immediate.

Now we prove the necessity. Suppose that $n=2^{\alpha}\left(2^{\alpha+1}-2^{r_{1}}-\cdots-2^{r_{k}}-1\right)$ is near-perfect, then from Proposition 1.5, there exists some $\alpha_{0}$, such that $0<\alpha_{0} \leqslant \alpha$, and

$$
2^{\alpha+1}-2^{r_{1}}-\cdots-2^{r_{k}}-1=2^{\alpha+1}-2^{\alpha_{0}}-1 ;
$$

or there exists some prime $q$, such that $\alpha=2 q-1$ and

$$
2^{\alpha+1}-2^{r_{1}}-\cdots-2^{r_{k}}-1=2^{q}-1 \text {; }
$$


or $\alpha=3$ and

$$
2^{\alpha+1}-2^{r_{1}}-\cdots-2^{r_{k}}-1=5 .
$$

From (I), we know that

$$
2^{\alpha_{0}-r_{k}}=2^{r_{1}-r_{k}}+\cdots+2^{r_{k-1}-r_{k}}+1 .
$$

The left side is even. From $r_{1}>r_{2}>\cdots>r_{k}$ we know that the right side is odd. This is a contradiction.

From (II), we have

$$
2^{q-r_{k}}=2^{2 q-r_{k}}-2^{r_{1}-r_{k}}-\cdots-2^{r_{k-1}-r_{k}}-1 \geqslant 0,
$$

thus $q \geqslant r_{k}$. From $2 q=\alpha+1>r_{1}>r_{2}>\cdots>r_{k}$ we know that the right side is odd, and so $q=r_{k}$. Hence

$$
1=2^{q-1}-2^{r_{1}-q-1}-\cdots-2^{r_{k-1}-q-1} .
$$

Now from $r_{k-1} \geqslant r_{k}+1=q+1$, the left side of the above equation is odd, and then $r_{k-1}=q+1$. Thus we can get $r_{k-2}=q+2, \ldots, r_{1}=q+k-1$. Then taking $2 q=\alpha+1, r_{k}=q, r_{k-1}=q+1, \ldots, r_{1}=q+k-1$ to the equation (II), we have

$$
2^{2 q}-2^{q+k-1}-\cdots-2^{q+2}-2^{q+1}-2^{q}=2^{q},
$$

i.e.,

$$
2^{2 q}-2^{q}\left(2^{k}-1\right)=2^{q},
$$

thus $k=q, r_{i}=2 q-i(1 \leqslant i \leqslant k)$.

From (III), we have

$$
2^{3}-2^{r_{1}-1}-\cdots-2^{r_{k}-1}=3
$$

thus $r_{k}=1$, and

$$
2^{r_{1}-1}+\cdots+2^{r_{k-1}-1}=4 .
$$

Note that $r_{1}>r_{2}>\cdots>r_{k}$, therefore $k-1=1, r_{1}=3$. In this case $n=40$.

Thus we complete the proof of Corollary 1.10 .

In the present paper, by Lemmas 1.7-1.8, we give two equivalent conditions of all even near-perfect numbers in the form $2^{\alpha} p_{1} p_{2}$ and $2^{\alpha} p_{1}^{2} p_{2}\left(p_{1}<p_{2}\right)$ (Theorem 2.1 and Theorem 3.1). These near-perfect numbers involve some special kinds of prime number pairs. One kind is exactly the new Mersenne conjecture's prime number pair. Another kind has the form $p_{1}=2^{\alpha+1}-1$ and $p_{2}=\frac{p_{1}^{2}+p_{1}+1}{3}$, where the former is a Mersenne prime and the latter's behavior is very much like a Fermat number. 


\section{Near-perfect numbers in the form $2^{\alpha} p_{1} p_{2}$}

Suppose that $n=2^{\alpha} p_{1} p_{2}$ is near-perfect, where $\alpha \geqslant 1$, and both $p_{1}$ and $p_{2}$ are odd primes with $p_{1}<p_{2}$. From Lemma 1.8, taking $r=2$ and $\alpha_{1}=\alpha_{2}=1$, we have that the redundant divisor $d$ is even. Hence $d=2^{\beta}, 2^{\beta} p_{1}, 2^{\beta} p_{2}$ $(1 \leqslant \beta \leqslant \alpha)$, or $2^{\beta} p_{1} p_{2}(1 \leqslant \beta \leqslant \alpha-1)$. Thus we obtain an equivalent condition of all even near-perfect numbers in the form $2^{\alpha} p_{1} p_{2}$. In fact we have the following result.

Theorem 2.1. Let $\alpha$ be a positive integer. Suppose that both $p_{1}$ and $p_{2}$ are odd primes with $p_{1}<p_{2}$. Then $n=2^{\alpha} p_{1} p_{2}$ is near-perfect if and only if one of the following conditions is true.

(1) $p_{1}=\frac{2^{\alpha+1}-1+k}{2^{\beta}+1-k}$, where $k=\frac{2^{\alpha+1}-1}{p_{2}}$ and $1 \leqslant \beta \leqslant \alpha-1$. In this case, the redundant divisor is $2^{\beta} p_{1} p_{2}$.

(2) $p_{1}=2^{\alpha+1}-1+\frac{2^{\alpha}-2^{\beta-1}}{k}$, where $k$ is determined by the equation $p_{2}=$ $\left(2^{\alpha+1}-1\right)(2 k+1)-2^{\beta}, 1 \leqslant \beta \leqslant \alpha$. In this case, the redundant divisor is $2^{\beta} p_{1}$.

(3) $p_{2}=2^{\alpha+1}-1+\frac{2^{2 \alpha+1}-2^{\alpha}-2^{\beta-1}}{k}$, where $k=\frac{p_{1}-\left(2^{\alpha+1}-1\right)}{2}$ and $1 \leqslant \beta \leqslant \alpha$. In this case, the redundant divisor is $2^{\beta}$.

Proof. We first prove the sufficiency.

(1) From the assumption we know that

$$
\left(2^{\beta}+1\right) p_{1}=\left(2^{\alpha+1}-1\right)+k+p_{1} k, 2^{\alpha+1}-1=p_{2} k .
$$

Note that $n=2^{\alpha} p_{1} p_{2}$, hence

$$
\begin{aligned}
\sigma(n)-2 n & =\left(2^{\alpha+1}-1\right)\left(p_{1}+p_{2}+1\right)-p_{1} p_{2} \\
& =\left(2^{\alpha+1}-1\right) \frac{2^{\beta}+1}{k} p_{1}-p_{1} \frac{2^{\alpha+1}-1}{k} \\
& =2^{\beta} p_{1} p_{2} .
\end{aligned}
$$

Note that $1 \leqslant \beta \leqslant \alpha-1$, thus $2^{\beta} p_{1} p_{2} \mid n$ and $2^{\beta} p_{1} p_{2} \neq n$. Therefore from the definition of the near-perfect number, $n=2^{\alpha} p_{1} p_{2}$ is near-perfect with the redundant divisor $2^{\beta} p_{1} p_{2}$.

The sufficiency proofs of (2) and (3) are similar.

Thus we complete the proof of the sufficiency.

Now we prove the necessity. Suppose that $n=2^{\alpha} p_{1} p_{2}$ is near-perfect with the redundant divisor $d$.

First, we can conclude that $d \neq 2^{\beta} p_{2}(1 \leqslant \beta \leqslant \alpha)$. Otherwise, from $d=2^{\beta} p_{2}$ and Lemma 1.7, we have

$$
p_{2} \mid \sigma\left(2^{\alpha} p_{1}\right)=\left(2^{\alpha+1}-1\right)\left(p_{1}+1\right) .
$$

Note that $p_{1}<p_{2}$, and then $\operatorname{gcd}\left(p_{2}, p_{1}+1\right)=1$, thus

$$
p_{2} \mid\left(2^{\alpha+1}-1\right) \text {. }
$$

Set $2^{\alpha+1}-1=k p_{2}$, then

$$
2^{\beta} p_{2}=d=\sigma(n)-2 n
$$




$$
\begin{aligned}
& =\left(2^{\alpha+1}-1\right)\left(p_{1}+1\right)\left(p_{2}+1\right)-2^{\alpha+1} p_{1} p_{2} \\
& =k p_{2}\left(p_{1}+1\right)\left(p_{2}+1\right)-\left(k p_{2}+1\right) p_{1} p_{2} \\
& =p_{2}\left[2^{\alpha+1}-1+(k-1)\left(p_{1}+1\right)+1\right] .
\end{aligned}
$$

Thus

$$
2^{\beta}=2^{\alpha+1}-1+(k-1)\left(p_{1}+1\right)+1 .
$$

But from $1 \leqslant \beta \leqslant \alpha$, we know that

$$
2^{\alpha+1}-1+(k-1)\left(p_{1}+1\right)+1 \geqslant 2^{\alpha+1}>2^{\beta},
$$

which is a contradiction.

Therefore the redundant divisor $d$ must be in the form

$$
2^{\beta}, 2^{\beta} p_{1}(1 \leqslant \beta \leqslant \alpha) \text { or } 2^{\beta} p_{1} p_{2}(1 \leqslant \beta \leqslant \alpha-1) .
$$

Now we prove the necessity of Theorem 2.1 according to the form of the redundant divisor $d$.

(1) Suppose that $d=2{ }^{\beta} p_{1} p_{2}(1 \leqslant \beta \leqslant \alpha-1)$, then from Lemma 1.7,

$$
p_{2} \mid \sigma\left(2^{\alpha} p_{1}\right)=\left(2^{\alpha+1}-1\right)\left(p_{1}+1\right) .
$$

Note that $\operatorname{gcd}\left(p_{2}, p_{1}+1\right)=1$, so

$$
p_{2} \mid\left(2^{\alpha+1}-1\right) .
$$

Set $2^{\alpha+1}-1=k p_{2}$, then

$$
\begin{aligned}
2^{\beta} p_{1} p_{2}=d & =\sigma(n)-2 n \\
& =k p_{2}\left(p_{1}+1\right)\left(p_{2}+1\right)-\left(k p_{2}+1\right) p_{1} p_{2},
\end{aligned}
$$

namely,

Therefore

$$
\left(2^{\beta}+1\right) p_{1}=k\left(p_{1}+p_{2}+1\right) .
$$

$$
p_{1}=\frac{2^{\alpha+1}-1+k}{2^{\beta}+1-k} .
$$

Thus we complete the proof of $(1)$.

(2) Suppose that $d=2^{\beta} p_{1}(1 \leqslant \beta \leqslant \alpha)$, then from Lemma 1.7 we have

$$
p_{1} \mid \sigma\left(2^{\alpha} p_{2}\right)=\left(2^{\alpha+1}-1\right)\left(p_{2}+1\right),
$$

thus

$$
p_{1} \mid\left(2^{\alpha+1}-1\right) \text {, or } p_{1} \mid\left(p_{2}+1\right) .
$$

If $p_{1} \mid\left(2^{\alpha+1}-1\right)$, we can set $2^{\alpha+1}-1=k p_{1}$, then

$$
\begin{aligned}
2^{\beta} p_{1}=d & =\sigma(n)-2 n \\
& =k p_{1}\left(p_{1}+1\right)\left(p_{2}+1\right)-\left(k p_{1}+1\right) p_{1} p_{2} \\
& =p_{1}\left[2^{\alpha+1}+(k-1)\left(p_{2}+1\right)\right],
\end{aligned}
$$

namely,

$$
2^{\beta}=2^{\alpha+1}+(k-1)\left(p_{2}+1\right) .
$$


Note that $1 \leqslant \beta \leqslant \alpha$, hence

$$
2^{\alpha+1}+(k-1)\left(p_{2}+1\right) \geqslant 2^{\alpha+1}>2^{\beta},
$$

which is a contradiction.

Therefore $p_{1} \nmid\left(2^{\alpha+1}-1\right)$, and so we must have $p_{1} \mid\left(p_{2}+1\right)$. Set $p_{2}=2 k p_{1}-1$, then from

$$
\begin{aligned}
2^{\beta} p_{1}=d & =\sigma(n)-2 n \\
& =\left(2^{\alpha+1}-1\right)\left(p_{1}+1\right) 2 k p_{1}-2^{\alpha+1} p_{1}\left(2 k p_{1}-1\right) \\
& =2 p_{1}\left[k\left(2^{\alpha+1}-1-p_{1}\right)+2^{\alpha}\right],
\end{aligned}
$$

we can get

$$
p_{1}=2^{\alpha+1}-1+\frac{2^{\alpha}-2^{\beta-1}}{k} .
$$

Thus we complete the proof of $(2)$.

(3) Suppose that $d=2^{\beta}(1 \leqslant \beta \leqslant \alpha)$, then $p_{2} \nmid d$. And so from Lemma 1.7 we have

$$
p_{2} \mid\left(\sigma\left(2^{\alpha} p_{1}\right)-d\right)=\left(2^{\alpha+1}-1\right)\left(p_{1}+1\right)-2^{\beta} .
$$

Set $\left(2^{\alpha+1}-1\right)\left(p_{1}+1\right)-2^{\beta}=2 k p_{2}$, i.e.,

$$
2 k p_{2}+2^{\beta}=\left(2^{\alpha+1}-1\right)\left(p_{1}+1\right) .
$$

Then

$$
\begin{aligned}
2^{\beta}=d & =\sigma(n)-2 n \\
& =\left(2 k p_{2}+2^{\beta}\right)\left(p_{2}+1\right)-2^{\alpha+1} p_{1} p_{2} \\
& =p_{2}\left[2 k\left(p_{2}+1\right)+2^{\beta}-2^{\alpha+1} p_{1}\right]+2^{\beta},
\end{aligned}
$$

hence

$$
2 k\left(p_{2}+1\right)+2^{\beta}-2^{\alpha+1} p_{1}=0 .
$$

And then by (2.1), we have

$$
p_{1}=2^{\alpha+1}-1+2 k, p_{2}=2^{\alpha+1}-1+\frac{2^{2 \alpha+1}-2^{\alpha}-2^{\beta-1}}{k} .
$$

Thus we complete the proof of (3).

By taking $\alpha=2 \beta-5, k=2^{\beta-2}+1$ in (1) of Theorem 2.1, and by supposing that both $p_{1}=\frac{2^{\beta-2}+1}{3}$ and $p_{2}=2^{\beta-2}-1$ are odd primes, then one can get the near-perfect number $2^{2 \beta-5} p_{1} p_{2}$ with the redundant divisor $2^{\beta} p_{1} p_{2}$. The primes $p_{1}$ and $p_{2}$ have the form of (3) and (2), respectively, in the following conjecture.

The New Mersenne Conjecture ([1]). If two of the following statements about an odd prime number $p$ are true, the third is also true.

(1) $p=2^{k} \pm 1$ or $p=2^{2 k} \pm 3$.

(2) $2^{p}-1$ is a prime number.

(3) $\frac{2^{p}+1}{3}$ is a prime number. 
Searching by computer (Setting $\alpha=2 \beta-5, k=2^{\beta-2}+1$ ), for $\alpha \leqslant 1000$ one can get exactly 8 even near-perfect numbers which satisfy (1) of Theorem 2.1 as follows:

$$
\begin{aligned}
2^{9} \cdot 11 \cdot 31, \quad 2^{13} \cdot 43 \cdot 127, \quad 2^{25} \cdot 2731 \cdot 8191, \quad 2^{33} \cdot 43691 \cdot 131071, \\
2^{37} \cdot 174763 \cdot 524287, \quad 2^{61} \cdot 715827883 \cdot 2147483647, \\
2^{121} \cdot 768614336404564651 \cdot 2305843009213693951, \\
2^{253} \cdot \quad 56713727820156410577229101238628035243 \\
\cdot \quad 170141183460469231731687303715884105727,
\end{aligned}
$$

and the corresponding $\beta=7,9,15,19,21,33,63$ and 129 .

For the case $(2)$ of Theorem 2.1 , when $1 \leqslant \alpha \leqslant 10$, there exist exactly 38 even near-perfect numbers; when $11 \leqslant \alpha \leqslant 20$, there exist exactly 148 even near-perfect numbers; when $21 \leqslant \alpha \leqslant 25$, there exist exactly 103 near-perfect numbers. In particular, if $3 \leqslant \alpha \leqslant 6$, then there exist exactly 7 even nearperfect numbers:

$$
\begin{gathered}
2^{3} \cdot 17 \cdot 101, \quad 2^{3} \cdot 19 \cdot 37, \quad 2^{3} \cdot 17 \cdot 67, \quad 2^{5} \cdot 67 \cdot 937, \\
2^{5} \cdot 79 \cdot 157, \quad 2^{5} \cdot 71 \cdot 283, \quad 2^{6} \cdot 131 \cdot 3929,
\end{gathered}
$$

and the corresponding $\beta=2,3,3,3,5,5$ and 3 .

Finally, for the case (3) of Theorem 2.1, when $\alpha<100$ and $k \leqslant 100$, there exist exactly 248 even near-perfect numbers. In particular, if $3 \leqslant \alpha \leqslant 5$, then there exist exactly 10 even near-perfect numbers:

$$
\begin{aligned}
& 2^{3} \cdot 17 \cdot 131, \quad 2^{3} \cdot 19 \cdot 73, \quad 2^{4} \cdot 43 \cdot 113, \quad 2^{5} \cdot 67 \cdot 1069, \quad 2^{5} \cdot 103 \cdot 163, \\
& 2^{5} \cdot 83 \cdot 263, \quad 2^{5} \cdot 79 \cdot 313, \quad 2^{5} \cdot 73 \cdot 463, \quad 2^{5} \cdot 71 \cdot 563, \quad 2^{5} \cdot 67 \cdot 1063,
\end{aligned}
$$

and the corresponding $\beta=3,3,3,3,5,5,5,5,5$ and 5 .

\section{Near-perfect numbers in the form $2^{\alpha} p_{1}^{2} p_{2}$}

Suppose that $n=2^{\alpha} p_{1}^{2} p_{2}$ is near-perfect, where $\alpha \geqslant 1$, and both $p_{1}$ and $p_{2}$ are odd primes with $p_{1}<p_{2}$. From Lemma 1.8 the redundant divisor $d$ is even, and so $d=2^{\beta}, 2^{\beta} p_{1}, 2^{\beta} p_{1}^{2}, 2^{\beta} p_{2}, 2^{\beta} p_{1} p_{2}(1 \leqslant \beta \leqslant \alpha)$ or $2^{\beta} p_{1}^{2} p_{2}$ $(1 \leqslant \beta \leqslant \alpha-1)$. Thus we obtain an equivalent condition of all even nearperfect numbers in the form $2^{\alpha} p_{1}^{2} p_{2}\left(p_{1}<p_{2}\right)$. In fact we have:

Theorem 3.1. Let $\alpha$ be a positive integer. Suppose that both $p_{1}$ and $p_{2}$ are odd primes with $p_{1}<p_{2}$. Then $n=2^{\alpha} p_{1}^{2} p_{2}$ is near-perfect if and only if one of the following is true.

(1) There exist some $\beta$ and $\gamma$, such that $1 \leqslant \beta \leqslant \alpha, 0 \leqslant \gamma \leqslant 2$, and the redundant divisor $d=2^{\beta} p_{1}^{\gamma}=\left(2^{\alpha+1}-1\right)\left(p_{1}^{2}+p_{1}+1\right)-k p_{2}$, where $k=$ $p_{1}^{2}-\left(2^{\alpha+1}-1\right)\left(p_{1}+1\right)$.

(2) There exists some $\beta$, such that $1 \leqslant \beta \leqslant \alpha$, and the redundant divisor $d=2^{\beta} p_{2}=\left(2^{\alpha+1}-1\right)\left(p_{2}+1\right)-2 k p_{1}$, where $2 k=p_{1} p_{2}-\left(2^{\alpha+1}-1\right)\left(p_{1}+p_{2}+1\right)$. 
(3) There exist some $\beta$ and $\gamma$, such that $1 \leqslant \beta \leqslant \alpha, 1 \leqslant \gamma \leqslant 2$, and $2^{\beta} p_{1}^{\gamma}=\left(2^{\alpha+1}-1\right)\left(p_{1}+1\right)+k-p_{1}^{2}$, where $k=\frac{\left(2^{\alpha+1}-1\right)\left(p_{1}^{2}+p_{1}+1\right)}{p_{2}}$. In this case the redundant divisor $d=2^{\beta} p_{1}^{\gamma} p_{2}$.

Proof. First, we prove the sufficiency.

(1) From the assumption we have

$$
\begin{aligned}
k p_{2}+d+k & =\left(2^{\alpha+1}-1\right)\left(p_{1}^{2}+p_{1}+1\right)+p_{1}^{2}-\left(2^{\alpha+1}-1\right)\left(p_{1}+1\right) \\
& =2^{\alpha+1} p_{1}^{2} .
\end{aligned}
$$

Note that $n=2^{\alpha} p_{1}^{2} p_{2}$, then

$$
\begin{aligned}
\sigma(n)-2 n & =\left(2^{\alpha+1}-1\right)\left(p_{1}^{2}+p_{1}+1\right)\left(p_{2}+1\right)-2^{\alpha+1} p_{1}^{2} p_{2} \\
& =\left(k p_{2}+d\right)\left(p_{2}+1\right)-2^{\alpha+1} p_{1}^{2} p_{2} \\
& =p_{2}\left[k\left(p_{2}+1\right)+d-2^{\alpha+1} p_{1}^{2}\right]+d \\
& =d,
\end{aligned}
$$

and $d=2^{\beta} p_{1}^{\gamma}(1 \leqslant \beta \leqslant \alpha, 0 \leqslant \gamma \leqslant 2)$, we can get $d \mid n$ and $d \neq n$. Thus from the definition we know that $n=2^{\alpha} p_{1}^{2} p_{2}$ is near-perfect with the redundant divisor $d$.

The sufficiency proofs of (2) and (3) are similar.

Now we prove the necessity. Suppose that $n=2^{\alpha} p_{1}^{2} p_{2}$ is near-perfect with the redundant divisor $d$.

(1) If $d=2^{\beta} p_{1}^{\gamma}(1 \leqslant \beta \leqslant \alpha, 0 \leqslant \gamma \leqslant 2)$, then $p_{2} \nmid d$. By Lemma 1.7 we have

$$
p_{2} \mid\left(\sigma\left(2^{\alpha} p_{1}^{2}\right)-d\right)=\left(2^{\alpha+1}-1\right)\left(p_{1}^{2}+p_{1}+1\right)-d .
$$

Set

$$
\left(2^{\alpha+1}-1\right)\left(p_{1}^{2}+p_{1}+1\right)-d=k p_{2} .
$$

Note that $n$ is near-perfect with the redundant divisor $d$, therefore

$$
\begin{aligned}
d & =\sigma(n)-2 n \\
& =\left[\left(2^{\alpha+1}-1\right)\left(p_{1}^{2}+p_{1}+1\right)-d\right]\left(p_{2}+1\right)+d\left(p_{2}+1\right)-2^{\alpha+1} p_{1}^{2} p_{2} \\
& =k p_{2}\left(p_{2}+1\right)+d\left(p_{2}+1\right)-2^{\alpha+1} p_{1}^{2} p_{2} \\
& =p_{2}\left[k\left(p_{2}+1\right)+d-2^{\alpha+1} p_{1}^{2}\right]+d,
\end{aligned}
$$

namely,

$$
k\left(p_{2}+1\right)+d-2^{\alpha+1} p_{1}^{2}=0,
$$

thus

By (3.1) we know that

$$
k p_{2}=2^{\alpha+1} p_{1}^{2}-k-d
$$

$$
2^{\alpha+1} p_{1}^{2}-k=\left(2^{\alpha+1}-1\right)\left(p_{1}^{2}+p_{1}+1\right),
$$

hence

$$
k=p_{1}^{2}-\left(2^{\alpha+1}-1\right)\left(p_{1}+1\right) .
$$


Thus we complete the proof of (1).

(2) If $d=2^{\beta} p_{2}(1 \leqslant \beta \leqslant \alpha)$, then $p_{1} \nmid d$. From Lemma 1.7 we have

$$
p_{1} \mid\left(\sigma\left(2^{\alpha} p_{2}\right)-d\right)=\left(2^{\alpha+1}-1\right)\left(p_{2}+1\right)-2^{\beta} p_{2} .
$$

Set

$$
\left(2^{\alpha+1}-1\right)\left(p_{2}+1\right)-2^{\beta} p_{2}=2 k p_{1},
$$

i.e.,

Thus from

$$
2^{\beta} p_{2}=\left(2^{\alpha+1}-1\right)\left(p_{2}+1\right)-2 k p_{1} .
$$

$$
\begin{aligned}
2^{\beta} p_{2}= & d \\
= & \sigma(n)-2 n \\
= & {\left[\left(2^{\alpha+1}-1\right)\left(p_{2}+1\right)-2^{\beta} p_{2}\right]\left(p_{1}^{2}+p_{1}+1\right)+2^{\beta} p_{2}\left(p_{1}^{2}+p_{1}+1\right) } \\
& -2^{\alpha+1} p_{1}^{2} p_{2} \\
= & 2 k p_{1}\left(p_{1}^{2}+p_{1}+1\right)+2^{\beta} p_{2} p_{1}\left(p_{1}+1\right)-2^{\alpha+1} p_{1}^{2} p_{2}+2^{\beta} p_{2} \\
= & p_{1}\left[2 k\left(p_{1}^{2}+p_{1}+1\right)+2^{\beta} p_{2}\left(p_{1}+1\right)-2^{\alpha+1} p_{1} p_{2}\right]+2^{\beta} p_{2},
\end{aligned}
$$

we know that

$$
2 k\left(p_{1}^{2}+p_{1}+1\right)+2^{\beta} p_{2}\left(p_{1}+1\right)-2^{\alpha+1} p_{1} p_{2}=0 .
$$

And then by (3.2),

$$
\left[\left(2^{\alpha+1}-1\right)\left(p_{2}+1\right)-2^{\beta} p_{2}\right]\left(p_{1}+1\right)+2 k+2^{\beta} p_{2}\left(p_{1}+1\right)-2^{\alpha+1} p_{1} p_{2}=0,
$$

hence

Therefore

$$
\left(2^{\alpha+1}-1\right)\left(p_{2}+1\right)\left(p_{1}+1\right)+2 k-2^{\alpha+1} p_{1} p_{2}=0 .
$$

namely,

$$
\left(2^{\alpha+1}-1\right)\left(p_{1}+p_{2}+1\right)+2 k-p_{1} p_{2}=0,
$$

$$
2 k=p_{1} p_{2}-\left(2^{\alpha+1}-1\right)\left(p_{1}+p_{2}+1\right) .
$$

Thus we complete the proof of (2).

(3) If $d=2^{\beta} p_{1}^{\gamma} p_{2}(1 \leqslant \beta \leqslant \alpha, 1 \leqslant \gamma \leqslant 2)$, then $p_{2} \mid d$. From Lemma 1.7 we have

$$
p_{2} \mid \sigma\left(2^{\alpha} p_{1}^{2}\right)=\left(2^{\alpha+1}-1\right)\left(p_{1}^{2}+p_{1}+1\right) .
$$

Set

$$
\left(2^{\alpha+1}-1\right)\left(p_{1}^{2}+p_{1}+1\right)=k p_{2} .
$$

Noting that

$$
\begin{aligned}
2^{\beta} p_{1}^{\gamma} p_{2} & =\sigma(n)-2 n \\
& =k p_{2}\left(p_{2}+1\right)-2^{\alpha+1} p_{1}^{2} p_{2},
\end{aligned}
$$

we have

$$
k\left(p_{2}+1\right)-2^{\alpha+1} p_{1}^{2}=2^{\beta} p_{1}^{\gamma} .
$$


Thus from (3.3) we know that

$$
\left(2^{\alpha+1}-1\right)\left(p_{1}^{2}+p_{1}+1\right)=2^{\alpha+1} p_{1}^{2}-k+2^{\beta} p_{1}^{\gamma},
$$

namely,

$$
2^{\beta} p_{1}^{\gamma}=\left(2^{\alpha+1}-1\right)\left(p_{1}+1\right)+k-p_{1}^{2} .
$$

Thus we complete the proof of $(3)$.

Corollary 3.2. Let $\alpha$ be a positive integer, and let both $p_{1}=2^{\alpha+1}-1$ and $p_{2}=p_{1}^{2}+p_{1}+1$ be odd primes. Then $n=2^{\alpha} p_{1}^{2} p_{2}$ is near-perfect if and only if $n=2 \cdot 3^{2} \cdot 13$.

Proof. From the definition of near-perfect numbers, the sufficiency is clear.

Now we prove the necessity. First, from Lemma 1.8, the redundant divisor $d$ of $n$ must have the form

$$
2^{\beta} p_{1}^{\gamma}(1 \leqslant \beta \leqslant \alpha, 0 \leqslant \gamma \leqslant 2), 2^{\beta} p_{2}(1 \leqslant \beta \leqslant \alpha),
$$

or

$$
2^{\beta} p_{1}^{\gamma} p_{2}(1 \leqslant \beta \leqslant \alpha, 1 \leqslant \gamma \leqslant 2) .
$$

If $d=2^{\beta} p_{1}^{\gamma}(1 \leqslant \beta \leqslant \alpha, 0 \leqslant \gamma \leqslant 2)$, then from (1) of Theorem 3.1 we have

$$
d=2^{\beta} p_{1}^{\gamma}=\left(2^{\alpha+1}-1\right)\left(p_{1}^{2}+p_{1}+1\right)-k p_{2} .
$$

Note that $p_{1}=2^{\alpha+1}-1$ and $p_{2}=p_{1}^{2}+p_{1}+1$, i.e.,

$$
2^{\beta} p_{1}^{\gamma}=p_{1} p_{2}-k p_{2}=\left(p_{1}-k\right) p_{2},
$$

thus $p_{2} \mid 2^{\beta} p_{1}^{\gamma}$, which is a contradiction to the fact that $\operatorname{gcd}\left(p_{2}, 2 p_{1}\right)=1$.

If $d=2^{\beta} p_{2}(1 \leqslant \beta \leqslant \alpha)$, then from (2) of Theorem 3.1 and the assumption, we have

$$
d=2^{\beta} p_{2}=\left(2^{\alpha+1}-1\right)\left(p_{2}+1\right)-2 k p_{1}=p_{1}\left(p_{2}+1-2 k\right),
$$

thus $p_{1} \mid 2^{\beta} p_{2}$, which is also a contradiction.

Therefore the redundant divisor $d$ must have the form $2^{\beta} p_{1}^{\gamma} p_{2}(1 \leqslant \beta \leqslant$ $\alpha, 1 \leqslant \gamma \leqslant 2$ ), namely, it satisfies (3) of Theorem 3.1. Thus we have

$$
d=2^{\beta} p_{1}^{\gamma} p_{2}=\left[\left(2^{\alpha+1}-1\right)\left(p_{1}+1\right)+k-p_{1}^{2}\right] p_{2},
$$

and

$$
k p_{2}=\left(2^{\alpha+1}-1\right)\left(p_{1}^{2}+p_{1}+1\right) .
$$

Note that $p_{1}=2^{\alpha+1}-1$ and $p_{2}=p_{1}^{2}+p_{1}+1$, so that

$$
2^{\beta} p_{1}^{\gamma} p_{2}=\left[p_{1}\left(p_{1}+1\right)+k-p_{1}^{2}\right] p_{2}=\left(k+p_{1}\right) p_{2}, k p_{2}=p_{1} p_{2},
$$

i.e., $k=p_{1}$ and $2^{\beta} p_{1}^{\gamma}=2 p_{1}$, which means that $\gamma=\beta=1$, and therefore $d=2 p_{1} p_{2}$.

On the other hand, since $p_{1}=2^{\alpha+1}-1$ is an odd prime number, we have $\alpha+1$ is prime. Set $q=\alpha+1$, then

$$
\begin{aligned}
p_{2} & =p_{1}^{2}+p_{1}+1=\left(2^{q}-1\right)^{2}+2^{q}=2^{2 q}-2^{q}+1 \\
& \equiv(-1)^{2 q}-(-1)^{q}+1 \quad(\bmod 3)
\end{aligned}
$$




$$
\equiv 2-(-1)^{q} \quad(\bmod 3) .
$$

If $q$ is an odd prime number, then $p_{2} \equiv 2+1 \equiv 0(\bmod 3)$, and thus $p_{2}=3$. This is a contradiction to the assumption $p_{2}=p_{1}^{2}+p_{1}+1>3$. Therefore $q=2$, i.e.,

$$
\alpha=1, p_{1}=3, p_{2}=13,
$$

which means that there exists a unique even near-perfect number $n=2 \cdot 3^{2} \cdot 13$, of the desired form, and the redundant divisor is $2 \cdot 3 \cdot 13$.

Thus we complete the proof of Corollary 3.2.

In the case (1) of Theorem 3.1, by taking

$$
\alpha=1, p_{1}=5, p_{2}=13, k=7,
$$

one can get a near-perfect number $n=2 \cdot 5^{2} \cdot 13$ with the redundant divisor $d=2$. Similarly, in (3) of Theorem 3.1 , by taking

$$
p_{1}=2^{\alpha+1}-1, p_{2}=\frac{p_{1}^{2}+p_{1}+1}{3}, k=3\left(2^{\alpha+1}-1\right), \beta=2, \gamma=1,
$$

when $p_{1}, p_{2}$ are prime, one can get the near-perfect number

$$
n=2^{\alpha}\left(2^{\alpha+1}-1\right)^{2} \cdot \frac{2^{2(\alpha+1)}-2^{\alpha+1}+1}{3},
$$

and the redundant divisor is

$$
d=2^{2}\left(2^{\alpha+1}-1\right) \cdot \frac{2^{2(\alpha+1)}-2^{\alpha+1}+1}{3} .
$$

In particular, in (3) of Theorem 3.1, by taking $q=\alpha+1=3,5,7,13$, respectively, then both $p_{1}=2^{q}-1$ and $p_{2}=\frac{2^{2 q}-2^{q}+1}{3}$ are odd primes. Thus one can get 4 near-perfect numbers as follows.

$2^{2} \cdot 7^{2} \cdot 19, \quad 2^{4} \cdot 31^{2} \cdot 331, \quad 2^{6} \cdot 127^{2} \cdot 5419, \quad 2^{12} \cdot 8191^{2} \cdot 22366891$.

Furthermore, searching by computer, $2^{60} \cdot\left(2^{61}-1\right)^{2} \cdot \frac{2^{122}-2^{61}+1}{3}$ is nearperfect satisfying the case (3) of Theorem 3.1. A natural question is if there are any other near-perfect numbers in this form, namely, does there exist a pair of primes in the form $\left(p_{1}=2^{\alpha+1}-1, p_{2}=\frac{p_{1}^{2}+p_{1}+1}{3}\right)$ ?

It is easy to see that $p_{1}$ must be a Mersenne prime. In fact, taking $p_{1}$ as one of the first twelve Mersenne primes (except the first one), we find that $p_{2}$ has no square factor (except 1). This is very much like Fermat numbers.

\section{References}

[1] P. T. Bateman, J. L. Selfridge, and S. S. Wagstaff Jr., The new Mersenne conjecture, Amer. Math. Monthly 96 (1989), no. 2, 125-128.

[2] R. P. Brent, G. L. Cohen, and H. J. J. te Riele, Improved techniques for lower bounds for odd perfect numbers, Math. Comp. 57 (1991), no. 196, 857-868.

[3] M. M. Buxton and S. R. Elmore, An extension of lower bounds for odd perfect numbers, Not. Amer. Math. Soc. 23 (1976), A-55.

[4] S. A. Fletcher, P. P. Nielsen, and P. Ochem, Sieve methods for odd perfect numbers, Math. Comp. 81 (2012), no. 279, 1753-1776. 
[5] G. H. Hardy and E. M. Wright, An Introduction to the Theory of Numbers, 6th Edition, Oxford Science Publishers, 2003.

[6] K. Ireland and M. Ira Rosen, A Classical Introduction to Modern Number Theory, 2nd Edition, Springer, 1990

[7] P. Ochem and M. Rao, Odd perfect numbers are greater than $10^{1500}$, Math. Comp. 81 (2012), no. 279, 1869-1877.

[8] P. Pollack and V. Shevelev, On perfect and near-perfect numbers, J. Number Theory 132 (2012), no. 12, 3037-3046.

[9] X. Z. Ren and Y. G. Chen, On near-perfect numbers with two distinct prime factors, Bull. Austral. Math. Soc. 88 (2013), no. 3, 520-524.

[10] W. Sierpiński, Sur les nombres pseudoparfaits, Mat. Vesnik 17 (1965), 212-213.

[11] N. J. Sloane, The online encyclopedia of integer sequences, accessible at http://oeis.org.

[12] B. Stubblefield, Greater lower bounds for odd perfect numbers, Env. Res. Lab. NOAA (1977), 209

[13] M. Tang, X. Z. Ren, and M. Li, On near-perfect and deficient-perfect numbers, Colloq. Math. 133 (2013), no. 2, 221-226.

YANBIN LI

Institute of Mathematics and Software Science

Sichuan Normal University

Sichuan Chengdu, 610066, P. R. China

QUNYING LIAO

Institute of Mathematics And Software Science

Sichuan Normal University

Sichuan Chengdu, 610066, P. R. China

E-mail address: qunyingliao@sicnu.edu.cn 